Supplementary information for

\title{
Direct measurement of crystal growth velocity in epitaxial phase-change material thin
} films

Mario Behrens*, Andriy Lotnyk*, Jürgen W. Gerlach, Martin Ehrhardt, Pierre Lorenz and Bernd Rauschenbach

Leibniz Institute of Surface Engineering (IOM), Permoserstr. 15, D-04318 Leipzig, Germany Email: mario.behrens@,iom-leipzig.de, andriy.lotnyk@iom-leipzig.de 

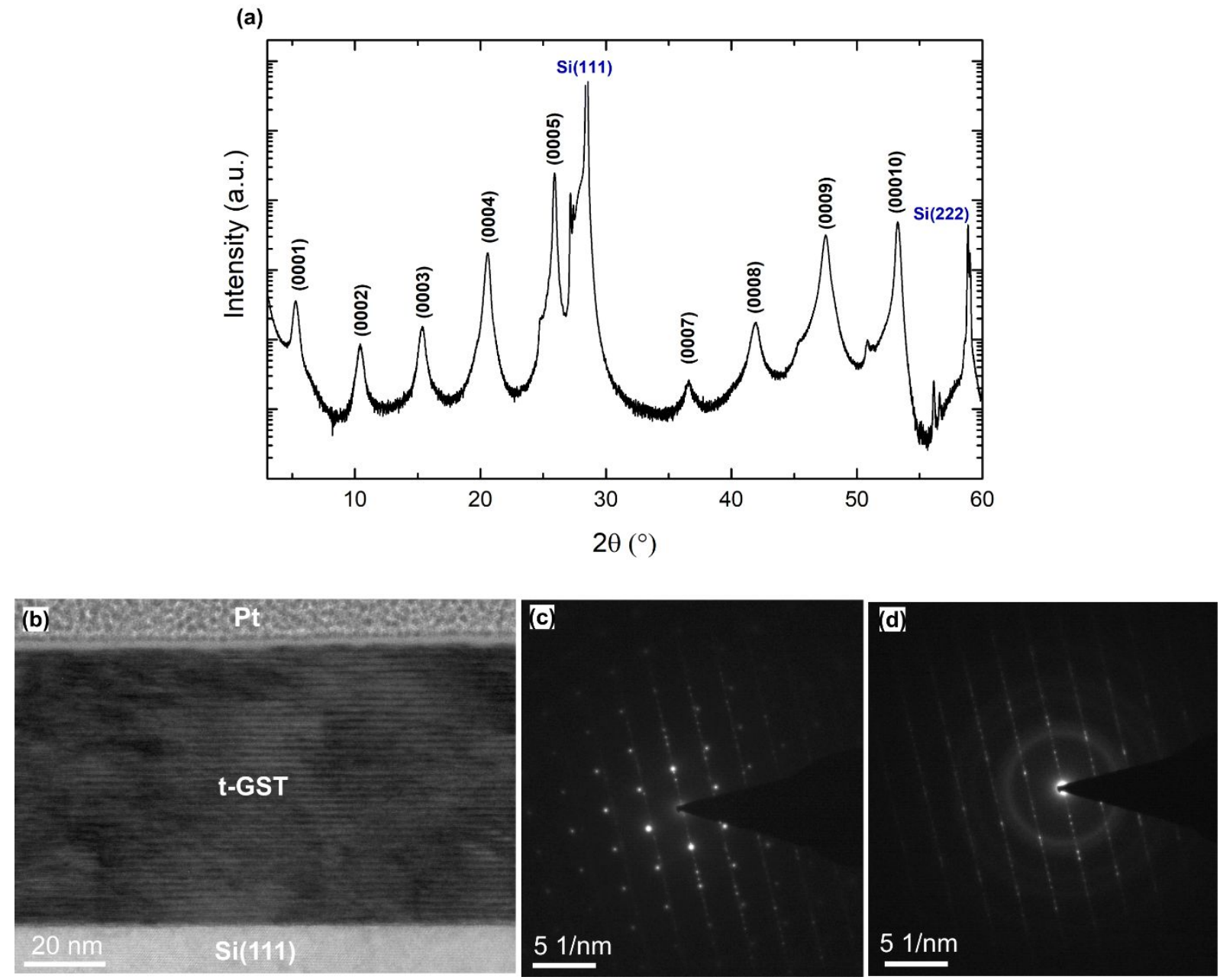

Figure S1: (a) x-ray diffraction $\theta-2 \theta$-pattern and (b) high-resolution transmission electron microscopy (HRTEM) image of an epitaxial trigonal $\mathrm{Ge}_{2} \mathrm{Sb}_{2} \mathrm{Te}_{5}$ (t-GST) thin film deposited on a $\mathrm{Si}(111)$ substrate. Selected area electron diffraction (SAED) pattern of (c) t-GST and Si confirming epitaxial relationship and (d) t-GST and Pt (ring pattern), where 5-fold periodicity of spot intensities reveals high structural ordering. 


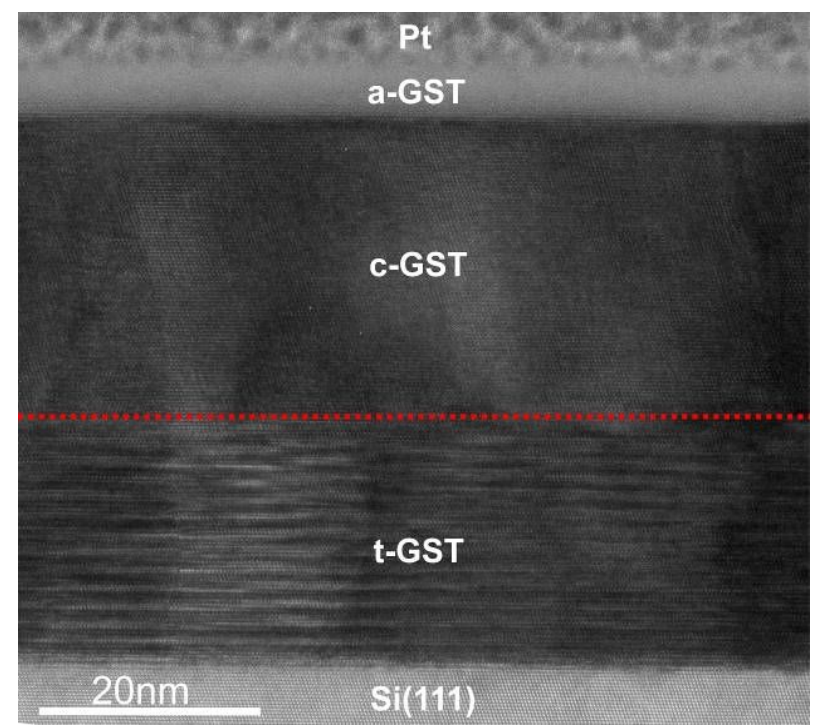

Figure S2: HRTEM image of the epitaxial t-GST thin film after application of four successive single ns-laser pulses of fluences of 35, 15, 35 and $15 \mathrm{~mJ} / \mathrm{cm}^{2}$. An epitaxial relationship between the initial t-GST and the recrystallized cubic GST (c-GST) layer is present 
(a) $22 \mathrm{~mJ} / \mathrm{cm}^{2}$

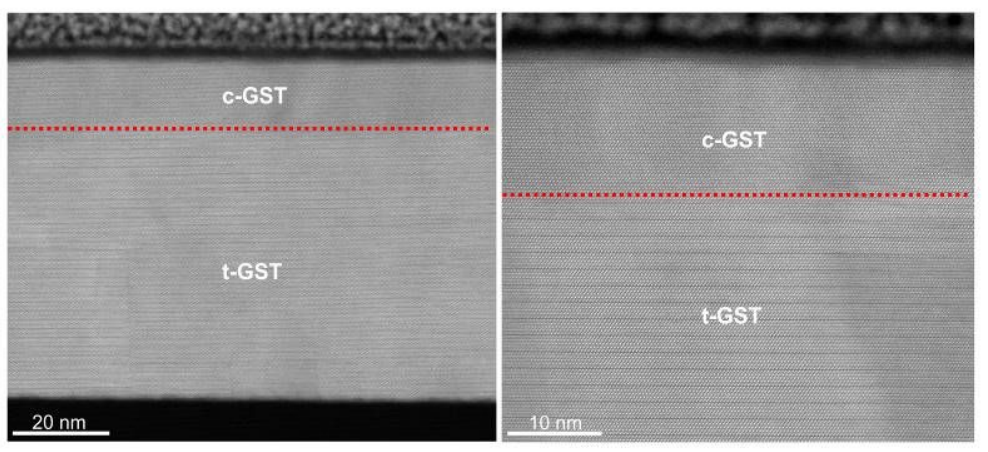

(b) $26 \mathrm{~mJ} / \mathrm{cm}^{2}$

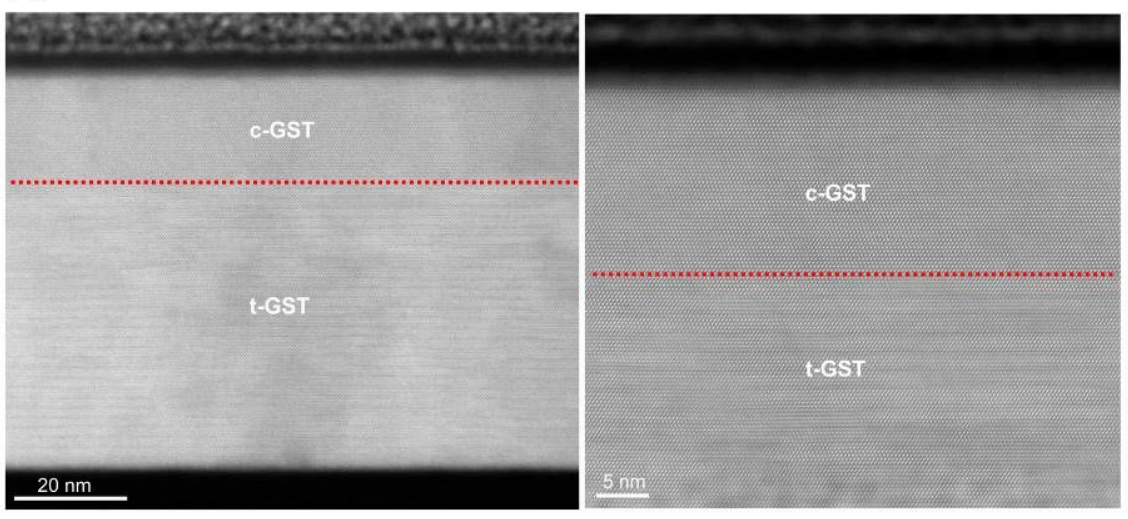

(c) $38 \mathrm{~mJ} / \mathrm{cm}^{2}$

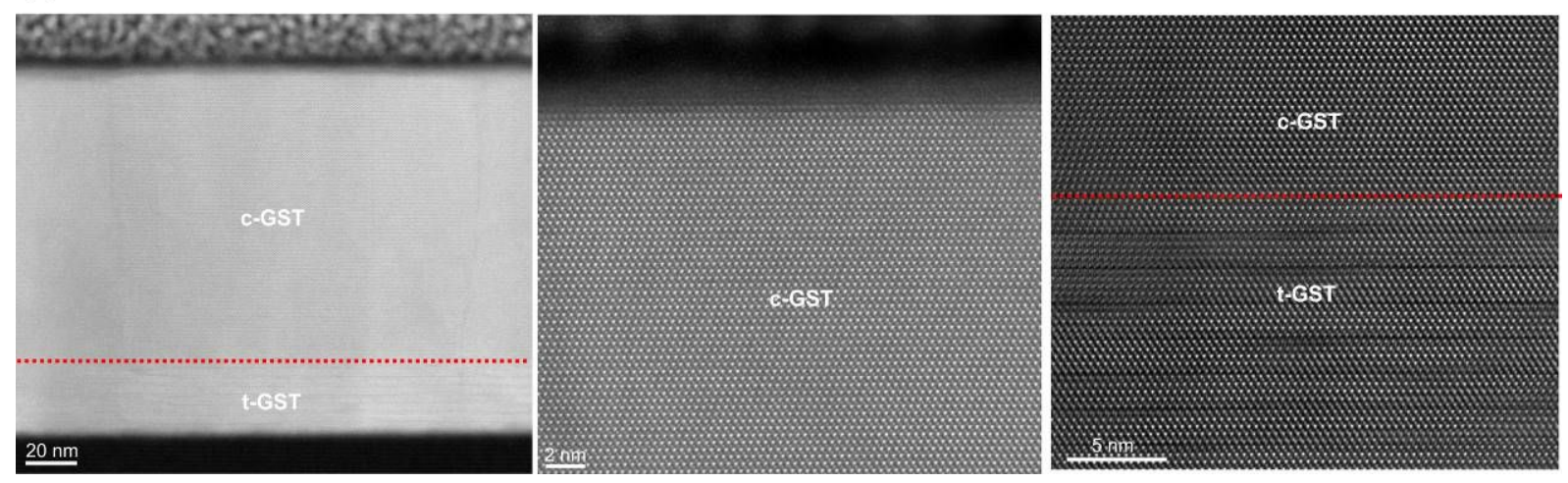

Figure S3: Selection of high-resolution scanning transmission electron microscopy (HRSTEM) images of the epitaxial t-GST thin film shown in figure 3 (corresponding reflectivity measurements shown in figure 1). The applied laser fluences are in figure (a) 22 $\mathrm{mJ} / \mathrm{cm}^{2}$, in figure (b) $26 \mathrm{~mJ} / \mathrm{cm}^{2}$ and in figure (c) $28 \mathrm{~mJ} / \mathrm{cm}^{2}$. The interfaces between $\mathrm{t}-\mathrm{GST}$ and c-GST layers are indicated by a dashed red lines. 

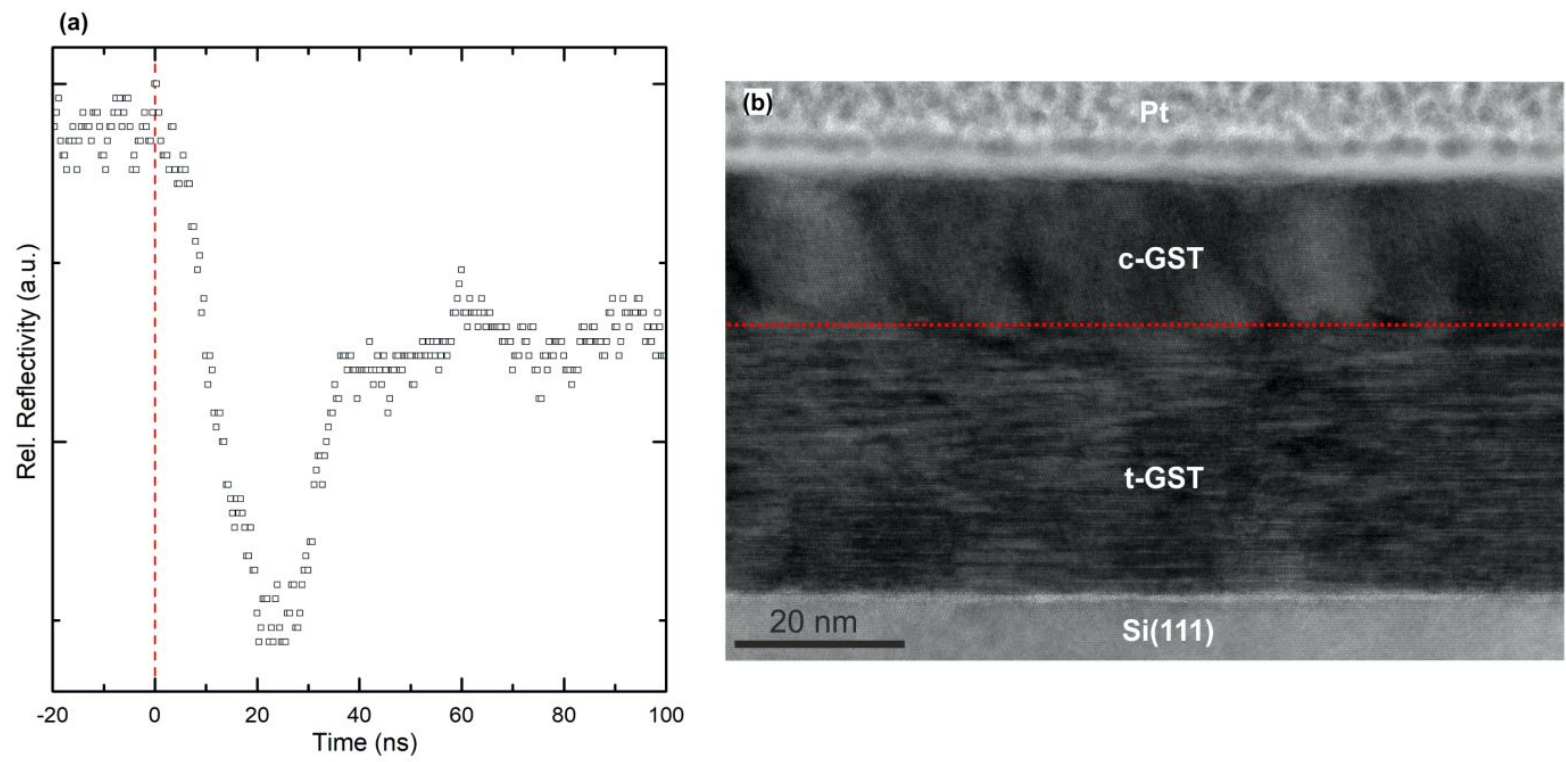

Figure S4: (a) time-resolved reflectivity and (b) HRSTEM results on single ns-laser pulse irradiation of a $50 \mathrm{~nm}$ thick epitaxial t-GST thin film. The thickness of the recrystallized cGST layer is $20 \mathrm{~nm}$. Considering a delay time of $\sim 4 \mathrm{~ns}$ after the $20 \mathrm{~ns}$ long laser pulse for crystallization to start, a crystal growth velocity of $1.4 \mathrm{~m} / \mathrm{s}$ is calculated. 